Journal for ImmunoTherapy of Cancer

\section{Rechallenge patients with immune checkpoint inhibitors following severe immune-related adverse events: review of the literature and suggested prophylactic strategy}

To cite: Haanen J, Ernstoff M, Wang Y, et al. Rechallenge patients with immune checkpoint inhibitors following severe immune-related adverse events: review of the literature and suggested prophylactic strategy. Journal for ImmunoTherapy of Cancer 2020;8:e000604. doi:10.1136/ jitc-2020-000604

Accepted 24 April 2020
Check for updates

(C) Author(s) (or their employer(s)) 2020. Re-use permitted under CC BY-NC. No commercial re-use. See rights and permissions. Published by BMJ.

For numbered affiliations see end of article.

Correspondence to

Dr Michel Obeid;

michel.obeid@chuv.ch

\section{ABSTRACT}

Patients with cancer who developed severe, grade 3 or 4 immune-related adverse events (irAEs) during therapy with immune checkpoint inhibitors are at risk for developing severe toxicities again on rechallenge with checkpoint inhibitors. Consequently, medical oncologists and multidisciplinary teams are hesitant to retreat in this scenario, despite the fact that a number of patients may derive clinical benefit from this approach. Balancing such clinical benefit and treatment-related toxicities for each patient is becoming increasingly challenging as more and more patients with cancer are being treated with checkpoint inhibitors. In this manuscript, we provide an extensive overview of the relevant literature on retreatment after toxicity, and suggest prophylactic approaches to minimize the risk of severe irAE following rechallenge with immune checkpoint blockade, since treatment may be lifesaving in a number of occasions.

\section{INTRODUCTION}

The number of patients with cancer who receive immune checkpoint-based therapies is rapidly increasing following a growing number of approved indications. Furthermore, these treatments are moving from later line therapies to front-line therapies in the metastatic setting, as well as entering the early-disease neoadjuvant and adjuvant field. Highly interesting results are emerging from immune checkpoint inhibition (ICI) in the neoadjuvant trials, ${ }^{1-4}$ as well as using combination strategies, for example, anti-CTLA-4 and anti-PD-(L)1, ICI plus other immunebased therapies, chemotherapy, radiation, antibody drug conjugates and targeted therapies. Parallel to this rise in number of patients treated with ICI, the number of patients developing immune-related adverse events (irAEs) is increasing. ${ }^{5}$ The incidence of severe toxicities differs depending on the agent administered, the dose, interval and duration of therapy. In descending order of risk, the highest risk of toxicity is seen with the combination of ipilimumab (anti-CTLA4) plus nivolumab (anti-PD-1) in metastatic melanoma, as in this disease patients are given high-dose ipilimumab $(3 \mathrm{mg} / \mathrm{kg})$ plus low-dose nivolumab $(1 \mathrm{mg} / \mathrm{kg})$ in melanoma and SCLC, low-dose ipilimumab of $1 \mathrm{mg} / \mathrm{kg}$ every 3 or 6 weeks plus nivolumab $3 \mathrm{mg} / \mathrm{kg}$ in renal cell carcinoma and non-small-cell lung carcinoma (NSCLC), or single-agent ipilimumab $(3 \mathrm{mg} / \mathrm{kg})$ in metastatic melanoma, or monotherapy anti-PD-1 or anti-PD-L1 agents in many indications.

These differences are also reflected by the discontinuation rate due to grade 3-4 irAEs (table 1). Most of these data are emerging from randomized controlled clinical trials, and may underestimate the discontinuation rate in 'real-world' setting. Importantly, centers participating in clinical trials with ICI are often more experienced in irAEs recognition and management.

In clinical trials, patients who develop severe toxicities on ICI-based therapies are usually not allowed to resume ICI once their disease progresses, because of the chance of developing severe irAEs on rechallenge with immunotherapies (majority with anti-PD-(L) one backbone combinations). Furthermore, a number of oncology teams may be hesitant to retreat patients or reinitiate ICI treatment even when the severe irAEs is under control and the immunosuppression is tapered off. This also depends on the type and grade of irAEs and how patients responded to the immunosuppressive treatment, and whether the patient is still on immunosuppressive 
Table 1 The rate of permanent discontinuation of different $\mathrm{ICls}$ because of treatment-related adverse events

\begin{tabular}{lll}
\hline Indication & Drug & $\begin{array}{l}\text { Discontinuation } \\
\text { rate, \% }\end{array}$ \\
\hline Metastatic & Ipilimumab & $14.8^{60}, 9.4^{61}$ \\
melanoma & Nivolumab & $7.7^{60}$ \\
& Ipilimumab+nivolumab & $36.4^{60}$ \\
& Pembrolizumab & $6.9^{61}$ (every 3 \\
& & weeks), $4^{61}$ (every \\
& & 2 weeks) \\
Metastatic & Ipilimumab+nivolumab & $22^{62}$ \\
renal cell & Nivolumab & $8^{63}$ \\
cancer & & \\
NSCLC & Pembrolizumab & $9^{64}$ \\
\hline
\end{tabular}

$\mathrm{ICl}$, immune checkpoint inhibition.

therapy to control the irAEs, as well as the availability of alternative therapy options. Another important element has recently been reported concerning the initial response to ICI before the onset of an irAEs. The efficacy in patients with NSCLC who had an objective response prior to the onset of an irAE was similar in the retreatment cohort and discontinuation cohort, thus, these patients may not benefit from ICI rechallenge. Nevertheless, among patients without objective response at the time of the first serious irAE, progression-free survival and overall survival (OS) were improved on retreatment compared with those in whom treatment remained discontinued. ${ }^{6}$ The major limitation of this study was the limited number of patients $(n=38)$.

Unfortunately, most risk-versus-benefit factors contributing to rechallenge decision are unknown, which makes the choice to restart (or not) immunotherapy very challenging.

In case of grade 4 cardiovascular or neurological/ muscular irAEs, or severe pneumonitis requiring admission to the intensive care unit, associated with a high mortality rate, we assume that the fear of both the oncology team and the patient to induce the same or more severe irAEs on retreatment is high. Moreover, for patients who require escalation of immunosuppression to two or more drugs (eg, high-dose corticosteroids (CS) plus either mycophenolate mofetil, tacrolimus or antithymocyte globulin (ATG)) to control the irAEs, we think the threshold to rechallenge with ICI will obviously get higher.

Thus, to choose between a potential effective treatment for a lethal cancer and Hippocrates 'adagium': 'primum non-nocere' (first, do no harm), can be very difficult. However, in order to make such clinical decisions, granular knowledge and careful balance of benefits and risks in a particular setting is needed. Here, we provide an overview of what is known in the literature and how we could use this knowledge to consider taking a calculated/balanced risk to possibly rechallenge a patient who recovered from severe irAEs with ICI-based therapy on a case by case basis. We propose a framework for a prophylactic immunosuppressive approach to minimize the risk of severe toxicities on retreatment and to more safely reintroduce ICI. Such decision should be systematically discussed and validated in the context of multidisciplinary team meeting according to each patient risk:benefit ratio and unique circumstances. These recommendations are based on experts' opinion. However, randomized clinical trials are needed to establish evidence-based guidelines forming the basis and criteria for ICI rechallenge in the future.

\section{Safety of retreatment with ICI after irAE resolution}

We see three scenarios in which reintroducing ICI is possible after development of severe irAEs: first, to opt for a class switch from anti-PD-(L) 1 to anti-CTLA-4 therapy or vice versa in diseases where both classes are clinically relevant; second, the rechallenge scenario with the reintroduction of the same class agent or the same molecule (ICI resumption) after (near) resolution of the irAE; and third, a secondary prevention scenario where ICI is resumed concomitantly with immunosuppressive therapy (table 2).

\section{Class switch}

So far, the only two classes of ICI in routine clinical use are anti-CTLA-4 and anti-PD-(L) 1. Anti-CTLA-4 works by increasing the diversity of the antitumor immune response and perhaps by depleting highly CTLA-4 expressing Tregs from the tumor microenvironment, whereas PD-1/PD-L1 blockade mostly works at the tumor site by locally re-activating a suppressed immune response. Because of these non-overlapping mechanisms of action, sequencing from one class to another may be feasible and beneficial for patients.

For the switch scenario, a retrospective analysis ${ }^{7}$ of 67 patients with metastatic melanoma evaluated the safety of anti-PD-1 in the setting of prior severe irAEs with ipilimumab. In this cohort, most patients experienced severe toxicity with ipilimumab $(76 \%$ grade $3,10 \%$ grade 4$)$, including $42(63 \%)$ patients with grade $3 / 4$ colitis (15 were treated with infliximab), $3(5 \%)$ with grade $3 / 4$ hepatitis (one of which required ATG) and 12 (18\%) with hypophysitis. All irAEs, except hypophysitis, had been resolved at the time of initiation of anti-PD-1. Interestingly, only two (3\%) patients had recurrence of the same irAEs with anti-PD-1 therapy. Twenty-three (34\%) patients developed new/different irAEs with anti-PD-1 therapy. Fourteen $(21 \%)$ patients had grade $3-4$ irAEs, and eight $(12 \%)$ discontinued therapy due to the development of grade $3 / 4$ pneumonitis $(n=4)$, hepatitis $(n=2)$, colitis $(n=1)$ and myasthenia gravis $(n=1)$. There were no treatment-related deaths. Of note, of the 12 patients with ipilimumab-induced hypophysitis, only one developed an irAEs, which was grade 3 colitis that was managed successfully with oral prednisone. With lack of prospective data, this retrospective study may suggest that the switch from anti-CTLA-4 to anti-PD-1 in patients with 


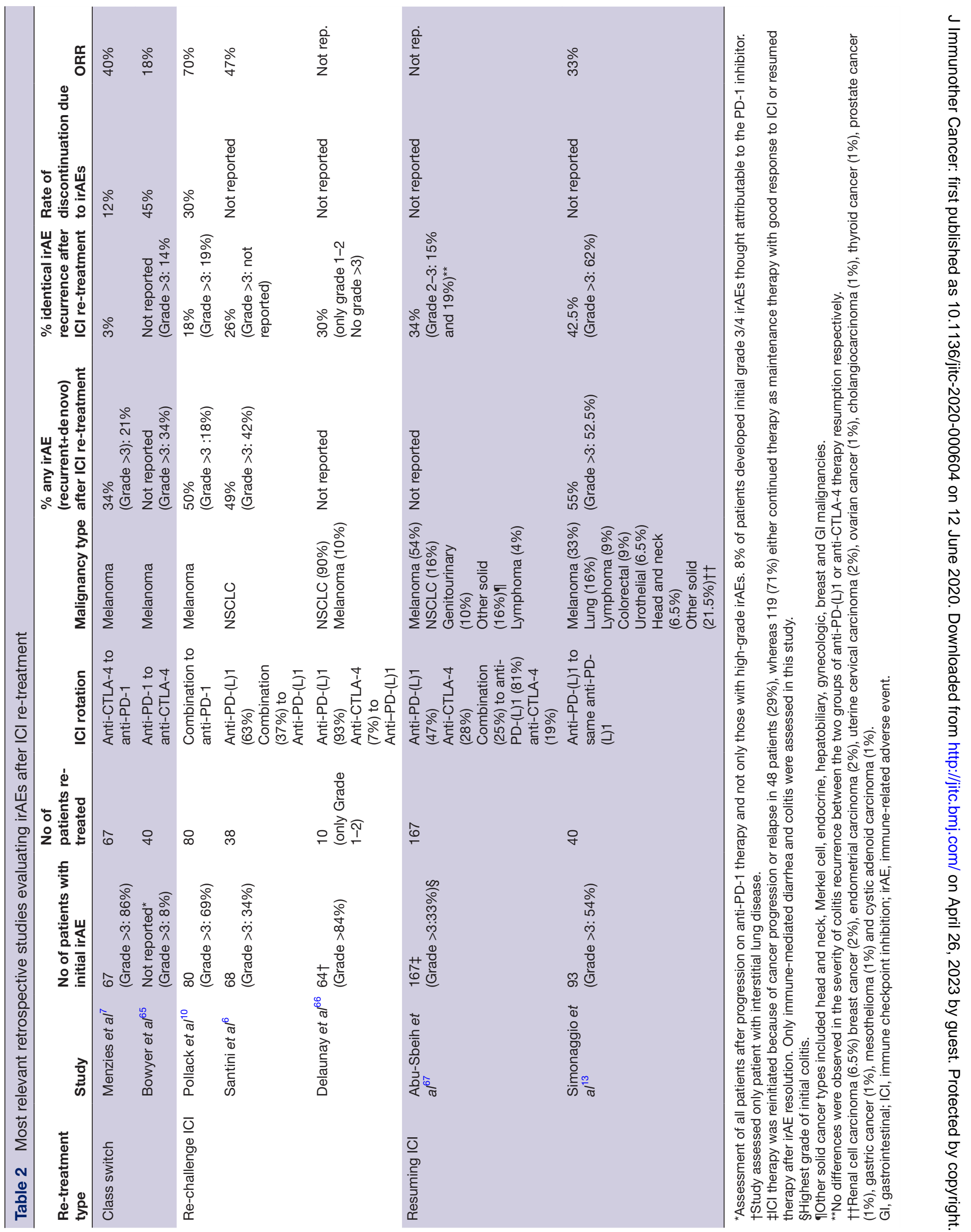


previous anti-CTLA-4-related severe irAEs may be feasible in a number of patients. Another retrospective study ${ }^{8}$ assessed the safety and toxicity of ipilimumab following failure of anti-PD- 1 therapy in 40 patients with metastatic melanoma (assessment of all patients after progression on anti-PD-1 therapy and not only those with high-grade irAEs). Initially $8 \%$ of patients developed grade $3 / 4$ irAEs attributable to the PD-1 inhibitor. Globally, 35\% of patients receiving ipilimumab after anti-PD-1 developed grade $3 / 4$ irAEs. In this cohort, diarrhea or colitis was the most common high-grade irAEs (23\%) with earlier onset, followed by hepatitis, pneumonitis and a case of encephalitis. In this cohort, three patients experienced grade $3 / 5$ pneumonitis, and one patient died due to this treatment complication. Importantly, pneumonitis has rarely been described with ipilimumab therapy and is more commonly reported with PD-1 inhibitors. Two of the $14(14 \%)$ patients who experienced high-grade irAEs with ipilimumab had developed severe irAEs (hypothyroidism, bullous pemphigoid) with previous anti-PD-1 therapy. Even with this seemingly increased frequency of high-grade ICI-related pneumonitis, we consider that the switch from anti-PD-1 to anti-CTLA4 therapy or from anti-CTLA4 to anti-PD-1 therapy may be considered, especially by an experienced team and in the absence of other available treatment options, and could be manageable in a number of cases.

The only prospective data available came from the CheckMate-064 study, ${ }^{9}$ in which patients with metastatic melanoma were randomized to receive either ipilimumab (four courses), followed by nivolumab (6 courses at 2-week intervals), or nivolumab followed by ipilimumab. Patients were evaluated at the end of second treatment at week 25. At that time point, there was not a significant difference in grade 3-4 irAEs between arms ( $50 \%$ in nivolumab >ipilimumab vs $43 \%$ in ipilimumab $>$ nivolumab). However, as expected, more patients developed grade 3-4 irAEs during the first induction treatment in the ipilimumab >nivolumabarm $(23 \%)$ vs $7 \%$ in patients receiving nivolumab during the first induction period. This was opposite in the second induction period with more grade 3-4 irAEs in the nivolumab $>$ ipilimumab-treated patients $(52 \%$ vs $28 \%)$. The most important reason to discontinue treatment was different between the two arms; toxicity versus disease progression were $34 \%$ and $26 \%$ for the nivolumab >ipilimumabarm and $17 \%$ and $56 \%$ for the ipilimumab $>$ nivolumab-treated patients, indicating that sequencing of ICI classes impacts on the discontinuation rate due to irAEs. All patients who started the first treatment, also initiated the second treatment. About $50 \%$ then went to maintenance nivolumab (both arms) after week 25, illustrating the feasibility of sequencing these drugs.

Importantly, when considering the sequencing from one class of ICI to another, the relatively long half-life of the agents and the duration of receptor occupancy of peripheral blood $\mathrm{T}$ cells, which can last for up to 2 months, can be important considerations. Therefore, on switching from one drug class to another within this period of receptor occupancy, may be equivalent to giving these agents combined with a possibly higher chance of inducing irAEs. Toxicity data from randomized controlled trials in patients treated with combination ICI upfront compared with sequential treatment is, however, not available.

\section{Re-challenge}

Another study ${ }^{10}$ of 80 patients retrospectively evaluated the safety of resuming anti-PD-1 monotherapy in patients with severe toxicity from ICI combination therapy leading initially to discontinuation. In this cohort, the patients discontinued combination therapy due to irAEs, including colitis $(41 \%)$, hepatitis $(36 \%)$ and pneumonitis $(4 \%)$. The highest grade of irAE experienced with combination therapy was grade 2 in $25(31 \%)$ patients, grade 3 in $49(61 \%)$ and grade 4 in $6(8 \%)$ patients. Among these patients, $77(96 \%)$ received CS and $21 \%$ received additional immunosuppression (eg, infliximab). All patients were rechallenged with anti-PD-1 and $40(50 \%)$ patients experienced any grade irAEs. Of these patients, 26 (33\%) had grade 1-2 events, and 14 (18\%) had grade 3-5 toxicities; 24 (30\%) patients discontinued anti-PD-1 due to these events; only 14 (18\%) had recurrent irAEs. Patients with neurological toxicity $(n=2)$, uveitis $(n=2)$ and ITP $(n=1)$ did not experience recurrence. On the contrary, hepatitis (5 recurrences out of 29 patients, $17 \%$ ), pancreatitis (2 of 2,100\%), pneumonitis (1 of $3,33 \%)$ and nephritis (1 of 2, 50\%) appeared to recur more often. Only one fatal Stevens-Johnson syndrome occurred after monotherapy rechallenge. Importantly, many of these toxicities were not confirmed by pathological examination of organ biopsies (or bronchoalveolar lavage (BAL) in case of pneumonitis), not excluding the potential for other causes of liver test abnormalities and creatinine elevation. Interestingly, colitis was less likely to recur compared with other irAEs (6\% vs $28 \%$ ). Clinically significant but distinct toxicities occurred in additional $17(21 \%)$ patients (11 grade $1-2$ and 6 grade $3-4)$. Ten of the 14 patients $(71 \%)$ discontinued anti-PD-1 therapy due to these recurrent irAEs, but in this relatively small series no other fatal events occurred. In total, 39\% ( $n=31$ ) of patients experienced clinically significant recurrent or distinct toxicities. Notably, the duration of steroid tapering, severity of initial irAEs and use of additional immunosuppressants did not predict toxicity on rechallenge in this retrospective analysis, although patients remaining on steroid therapy at the time of anti-PD-1 resumption had higher toxicity rates $(55 \%$ vs $31 \%)$. We noted a relatively high rate $(21 \%)$ of clinically significant but distinct irAEs on anti-PD-1 rechallenge that is higher than that the incidence noted with conventional single-agent anti-PD-1 therapy. In this study, the type of toxicity appeared to be informative. Very few patients with 'ipilimumab-like' toxicities, including colitis and hypophysitis, experienced recurrence with anti-PD-1 in contrast to 'anti-PD-1-like' toxicities, such as hepatitis, nephritis, 
pancreatitis and pneumonitis, which appeared to have higher risk of recurrence. Based on this small study, it can be considered that patients with previous ICI-related colitis or hypophysitis may potentially resume anti-PD-1 treatment after careful evaluation and discussion.

For the rechallenge scenario with the same agent class, a cohort ${ }^{6}$ of 38 patients with NSCLC who had experienced irAEs (34\% grade3-4) with anti-PD-(L) 1 (nivolumab, pembrolizumab, atezolizumab or durvalumab), either as monotherapy or in combination with anti-CTLA-4 therapy (ipilimumab or tremelimumab), were retreated with anti-PD-(L) 1 after irAE resolution or improvement to grade 1. In this study, after retreatment, 18 (48\%) patients had no subsequent irAEs, $10(26 \%)$ experienced recurrence of the initial irAE and $10(26 \%)$ had a new irAE. Most recurrent/new irAEs were mild (58\%) grade $1-2)$ and manageable (84\% resolved or improved to grade 1$)$. The rate of recurrent/new irAEs was comparable among patients who initially had grade $1 / 2$ or grade $3 / 4$ events (12 of $25(48 \%)$ vs 8 of $13(61 \%)$ ). Recurrent/ new irAEs were more common among those requiring hospitalization for the initial irAEs (7 of $8(87 \%)$ vs 13 of $30(43 \%))$. No evident trends in differential rates of new/recurrent irAEs based on the type of initial irAE were observed, except for higher frequency of recurrent/ new irAEs in patients who initially had arthralgia/myalgia (4 of 6, 67\%). Most recurrent/new irAEs in the retreatment cohort were mild (12 (60\%) grade $1-2 ; 8(40 \%)$ grades $3-4)$ and were manageable $(17(85 \%)$ resolved or improved to grade 1). Two treatment-related deaths occurred. Apart from the small size of the cohort, and the retrospective design of the study, another important limitation of this cohort was the absence of available information on the exact ICI used for the rechallenge. In summary, based on the limited available literature, we may consider rechallenge with ICI following temporary discontinuation because of grade 3-4 irAEs on prior ICI treatment, to be feasible in the majority of patients. We may expect new and/or recurrent toxicities to occur, the majority of which can be manageable, especially when given in centers with ample multi-disciplinary experience in irAEs management.

For ICI resumption, two recent studies revealed the feasibility of resuming ICI in patients who discontinue treatment due to irAEs. The first is a cohort ${ }^{11}$ of 93 patients who experienced $\geq$ grade 2 irAEs after anti-PD-(L) 1 had an anti-PD-(L) 1 rechallenge. In this cohort, the initial irAEs rates were $46 \%$ grade $2,39 \%$ grade $3 \%$ and $15 \%$ grade 4 events. In that study, 49 patients (53\%) had been treated with steroids, and 7 patients $(7.5 \%)$ required disease modifying or other immunosuppressive agents. Forty $(43 \%)$ of the 93 patients were rechallenged with the same ICI. Eighteen patients $(45 \%)$ did not experience further irAEs. However, 17 patients (42.5\%) experienced recurrence of the same type of irAE, and 5 patients (12.5\%) experienced a different irAE. Importantly, the frequency of recurrence irAEs differed: five out of six patients for arthralgia, two out of three for grade 4 neutropenia, three out of five for colitis, three out of five for hepatitis, three out of seven for skin irAEs, one out of five for pneumonitis and none of three for lipase elevation. The severity distribution for the second irAE was $38 \%$ for grade 2, $48 \%$ for grade 3 and $14 \%$ for grade 4 . The second irAEs were not more severe than the initial event. The second publication reported a study ${ }^{12}$ that evaluated 167 patients with ICI-related immune-mediated diarrhea and colitis (IMDC) recurrence after ICI resumption (the majority with the same class). Initial ICI therapy was PD-(L) 1 inhibitor in $47 \%$ of patients, CTLA- 4 inhibitor in $28 \%$ or a combination in $25 \%$ of patients. Seventy-five patients had an endoscopic evaluation, meaning that in $25 \%$ of patients the diagnosis IMDC and severity thereof (ulceration, extent of colon affected) was not present. Most patients who developed IMDC required immunosuppression with CS (113; 68\%); among them, 24 patients (14\%) required treatment escalation to addition of infliximab or vedolizumab (VDZ). Non-gastrointestinal (GI) irAEs were reported in 72 patients $(43 \%)$ and involved skin $(n=22)$, liver $(n=11)$, endocrine organs $(n=29)$, lung $(n=9)$ or other sites $(\mathrm{n}=19)$. Of the 167 patients in this cohort, 32 resumed CTLA-4 therapy, and 135 resumed anti-PD-(L) 1 therapy. IMDC recurred in 57 patients (34\%), with $44 \%$ on anti-CTLA- $4 \%$ and $32 \%$ on anti-PD-(L) 1 ; additionally, 47 of those patients $(82 \%)$ required immunosuppressive therapy, and all required permanent discontinuation of ICI. Most patients (70\%) developed grade 2 diarrhea, with $54 \%$ experiencing grade 1 colitis, and there was no irAE-related mortality.

Based on these case series, we have reason to believe that the IMDC severity after restarting ICI treatment can be manageable, and recurrence maybe less frequent after resumption of anti-PD-(L) 1 .

In making a decision on resuming the ICI, we have to take the original IMDC severity in careful consideration, as this will be different for patients with grade 4 IMDC requiring more than one drug to manage the colitis, compared with patients with mild colitis, which is more often the case with anti-PD-(L)1. Curiously, multivariable analysis in a study by Wang and team showed that patients treated with initial anti-CTLA-4 were significantly less likely to develop recurrent IMDC on rechallenge than those treated with anti-PD-(L) 1 treatment first. In contrast, the risk for IMDC recurrence in this study was significantly lower when PD-(L) 1 rather than CTLA-4 inhibitors were used for ICI resumption. That study also reported a significantly increased risk for IMDC recurrence with longer duration of IMDC symptoms and use of immunosuppressive therapy in the initial episode, associations that were also identified by Lambotte et $a l^{13}$, although without reaching statistical significance. The only factor significantly and independently linked with irAE recurrence in the Lambotte $e t$ al study was the time between treatment initiation and the first irAE: patients who did versus did not experience recurrence had a shorter such median interval at 9 and 15 weeks, respectively. 


\section{Rechallenge with concurrent immunosuppression?}

For the third scenario, available data are still very limited, but interesting reports have been published. In a study with 14 patients with initial severe ICI-related colitis, ICI was resumed after IMDC resolution. Eight patients received VDZ concurrently with ICI infusions, and six did not. Interestingly, after ICI resumption, the rate of IMDC recurrence with VDZ was significantly lower compared with that in patients without VDZ $(12.5 \%$ vs $50 \%$, respectively). Additionally, this rate obtained with secondary prevention was significantly lower than the rate reported previously in other studies without prevention estimated at approximately $35 \%-40 \% .{ }^{14}$ Another small study evaluated the concurrent therapy with ICI and tumor necrosis factor $\alpha(\mathrm{TNF} \alpha)$ blockade in patients with GI irAEs. All five patients tolerated further ICI with no recurrence of symptoms and repeat endoscopies showed resolution of acute inflammation and restaging imaging showed no cancer progression. ${ }^{15}$ Another retrospective study found that the use of prophylactic budesonide in patients with only microscopic colitis without visible endoscopic inflammation to be effective in allowing concomitant ICI therapy. ${ }^{16}$

Although no firm conclusions can be drawn from these three small studies, VZB clearly has activity in IMDC. Especially in the light of the recently published 'real-world' data on the potential decrease in survival of patients receiving infliximab as escalated immunosuppression for severe irAEs (especially IMDC) ${ }^{17}$ VZB maybe a reasonable alternative. However, given the underlying mechanism of action, potential negative impact from VZB in cancer response and outcome especially in patients with primary GI malignancy and GI involvement of distal metastasis from other primary cancers still requires further elucidation.

Moreover, OS analysis indicated no negative impact for VDZ adjunction. ${ }^{18}$ Another patient with metastatic melanoma and prior serious ICI-related arthritis and colitis received ipilimumab while remaining on tocilizumab TCZ. After 3 months of concomitant therapy with TCZ and ipilimumab, the patient's joint symptoms improved, and no symptoms of colitis/diarrhea were reported, despite being off budesonide. ${ }^{19}$ The concurrent introduction of selective immunosuppressants SIs such as VDZ or TCZ merit further investigation in prospective clinical trials as secondary prevention after ICI resumption in patients with previous severe irAEs to assess both oncological and irAE outcomes.

A conclusion from all these studies is the absence of reliable predictive and prognostic factors for severe recurrent or distinct irAEs after ICI readministration. Moreover, the risk factors are not clearly understood and are variable and inconsistent across studies. The small number of patients on which these studies are based, limited our ability to draw any formal conclusions and recommendations in terms of ICI rechallenge and secondary prevention strategies. However, in a number of patients with severe initial irAEs, ICI resumption could be considered, especially in the absence of therapeutic alternatives. However, in such cases, treatment decisions should be made based on an interdisciplinary expertize basis, also taking into account irAE type, grade and timing, response to immunosuppression, life expectancy, performance status, comorbid conditions, patient preferences, other available cancer therapy options, among other factors. In routine practice, ICI permanent discontinuation is often selected in patients with severe irAEs. We further advocate that great caution is needed regarding rechallenge. Actually, rechallenge should ideally be attempted with single agent ICI, only in patients with not life threatening, immunosuppression-sensitive and ideally resolved (or at least well controlled) initial irAEs. In contrast, occurrence of severe cardiovascular, neurological/muscular or other really threatening irAEs should raise even higher concern regarding ICI reexposure. Before ICI resumption, we highly advise for a personalized baseline assessment as proposed by Martins et $a l^{20}$ according to the risk profile of each patient, ideally by a multispecialty team in an experienced center. After potential ICI resumption, we advise very close monitoring for progression, prompt recognition and management of recurrent/distinct irAEs, as well as prompt ICI discontinuation as clinically indicated.

\section{Suggested approach to ICI rechallenge}

The correlation between irAEs and treatment response has been consistently reported for different cancer types ${ }^{21}$ such as melanoma, ${ }^{22-24}$ lung cancer ${ }^{25-28}$ or urothelial carcinoma $^{29}$; although few reports oppose such association. ${ }^{30}$ Importantly, one flaw with these studies is that patients developing irAEs may have received longer ICI treatment as patients with rapid disease progression may not have had the time to develop irAEs. Therefore, selection and confounding biases may have been introduced, and thus these results should be interpreted cautiously. The ICI resumption treatment after high-grade irAEs is always a challenging decision because of the potential severity of the irAE recurrence and the absence of formal guidelines. Given the reports of benefit from ICI resuming treatment in selected patients despite initial toxicity, it becomes an attractive option in certain patient populations.

Tumor-associated B cells in melanoma are involved in drug resistance and retain a protumorigenic property in part through IGF-1 secretion. ${ }^{31} \mathrm{CD} 20$ is also aberrantly expressed in subsets of melanoma cells with stemcell properties. ${ }^{32}$ A pilot study showed median survival exceeding 1 year in patients with metastatic melanoma receiving rituximab. ${ }^{33}$ Reassuringly, a recent report showed that B-cell depletion or absence had no effect on tumor growth, response to PD-1 inhibition or survival. ${ }^{34}$

Several reports showed major predominant CD8 $+\mathrm{T}$ cells infiltrate in different series of ICI-related myocarditis, ${ }^{35}{ }^{36}$ hepatitis $^{37}$ and acute interstitial nephritis (AIK) ${ }^{38}$ Recently, the analysis of BAL samples collected from patients with ICI-related pneumonitis showed increased BAL lymphocytosis, predominantly CD4 +T cells with central memory $\mathrm{T}$ cells with type I polarization 


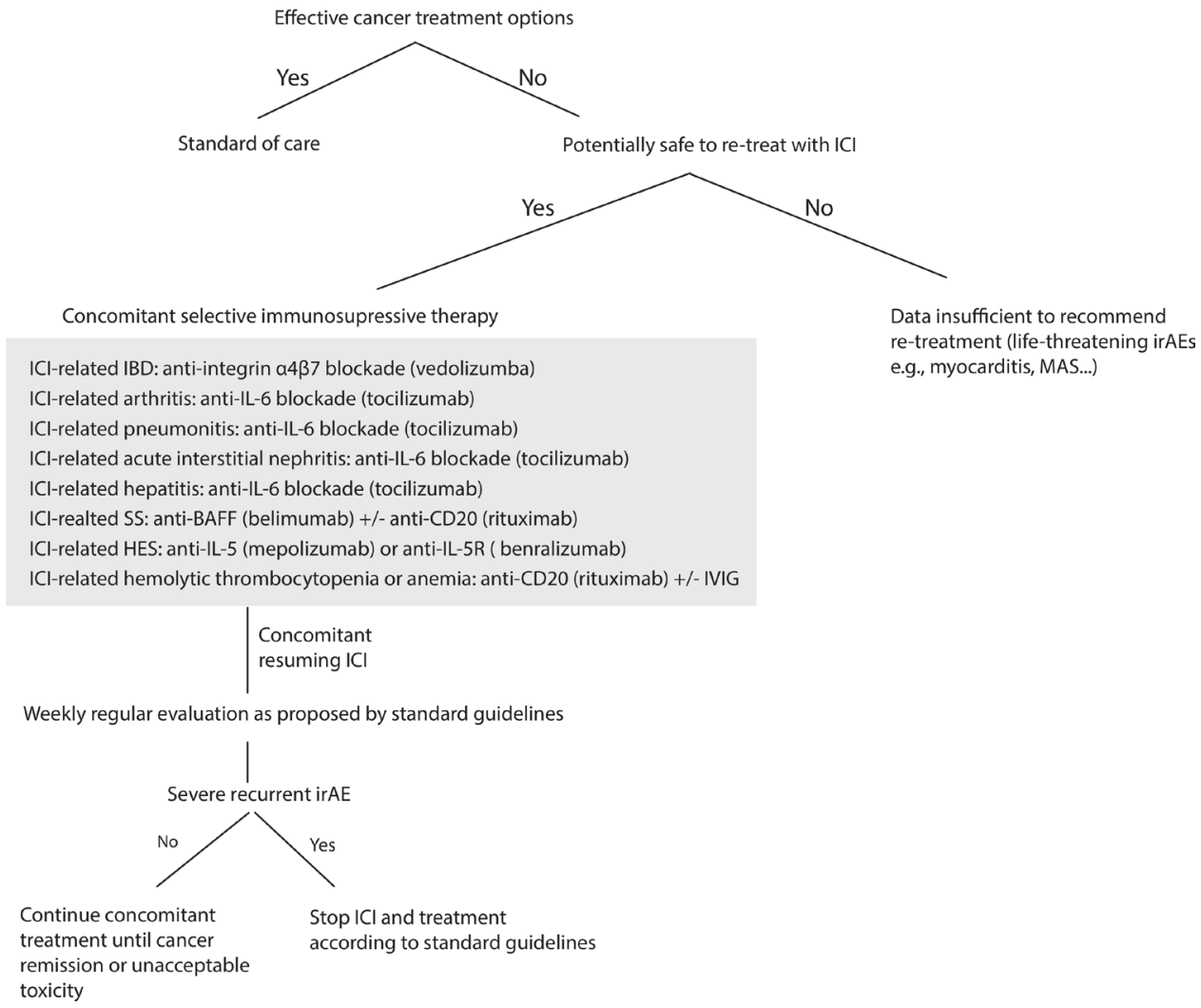

Figure 1 Flow chart for a suggested secondary prevention for ICl resumption after severe irAEs. For patients with no effective cancer treatment options and if there is no major contraindication related to a life-threatening irAEs, ICl could be resumed concomitantly with a prophylactic selective immunosuppressive therapy (SI). For severe ICl-related colitis, we advocate resuming ICl under VDZ, for severe ICI-related SS under anti-BAFF inhibitor \pm anti CD20 depletion, for severe ICI-related arthritis irAEs under TCZ, for ICl-related hemolytic anemia and thrombocytopenia irAEs under anti-CD20 depletion \pm IVIG. For ICI-related HES under anti-IL5(R), ICI-related pneumonitis under TCZ, ICl-related hepatitis under TCZ and ICl-related acute interstitial nephritis under TCZ. If patients experienced new or recurrent high-grade irAEs despite concomitant SI, we advocate the definitive $\mathrm{ICl}$ discontinuation and consideration of prompt local expertize for appropriate immunosuppressive treatment. VDZ $300 \mathrm{mg}$ once every 2 months, belimumab (BLM) at $10 \mathrm{mg} / \mathrm{kg}$ every 4 weeks, TCZ $162 \mathrm{mg}$ subcutaneously once per week or biweekly; or intravenously at $8 \mathrm{mg} / \mathrm{kg}$ once per month, RTX $500 \mathrm{mg}$ to $1000 \mathrm{mg}$ biweekly for $2 \mathrm{x}$, mepolizumab $300-750 \mathrm{mg}$ monthly and benralizumab at $30 \mathrm{mg}$ once monthly. BAFF, B-cell activating factor; HES, hypereosinophilic syndrome; IBD, inflammatory bowel disease; ICI, immune checkpoint inhibitor; IL-5, interleukin-5; irAEs, immune-related adverse events; IVIG, intravenous immunoglobulin; MAS, macrophage activation syndrome; PMR, polymyalgia rheumatica; RTX, rituximab; SS, Sjögren syndrome; TCZ, tocilizumab; VDZ, vedolizumab.

and decreased expression in BAL Tregs. ${ }^{40}$ Collectively, the data underline the essential and major role of $\mathrm{T}$ cells in the ICI-related toxicity.

Interleukin-6 (IL-6) is a major acute and chronic inflammatory phase mediator with protumorigenic properties. ${ }^{41}{ }^{42}$ During inflammation, IL-6 signaling drives T cell survival, expansion and proliferation. ${ }^{43}$ Intriguingly, blocking the IL-6 and PD-1/PD-L1 axes simultaneously provides synergistic antitumor effects on multiple levels, and the lack of IL- 6 in the tumor microenvironment augments Th1 immunity and increases the efficacy of ICI. $^{44-46}$ IL-17A-expressing CD $4^{+} \mathrm{T}$ cells $\left(\mathrm{c}-\mathrm{Kit}^{-} \mathrm{CD} 161^{+}\right.$ $\mathrm{MDR}^{+} \mathrm{Th} 17$ cells) have been found to be a major player of immune-related inflammation refractory to glucocorticoids. ${ }^{47}$ The pathogenic effect of IL-6 is indispensable in the differentiation of pro-inflammatory Th-17 cells from naive $\mathrm{CD} 4^{+} \mathrm{T}$ cells, suggesting an implication for Th17 subset in steroid-refractory irAEs. ${ }^{41}$ In contrast to other systemic immunosuppressive drugs (anti-TNF- $\alpha$ or nSI), blocking IL-6 is particularly interesting given this triple advantage without potentially compromising the efficacy of ICI. Similarly, it was also reported that interleukin- $1 \beta$ inhibition with canakinumab could significantly reduce incident lung cancer and lung cancer mortality. ${ }^{48}$

$\mathrm{VDZ}$ is a humanized monoclonal $\mathrm{IgG}_{1}$ antibody against $\alpha 4 \beta 7$ integrin that is approved for inflammatory bowel disease. ${ }^{490} \alpha 4 \beta 7$ integrin is expressed on a subset of CD4 $+\mathrm{T}$ cells that mediates homing of these cells specifically to the GI tract, hence allowing selective GI immunosuppression with $\mathrm{VDZ}^{51}$ while the immunity in extraintestinal tissues remains intact. ${ }^{52}$ In addition, VDZ does not bind to other integrins, such as $\alpha 4 \beta 1$, which is important for the homing of lymphocytes to a number of organs. ${ }^{52}$

Interestingly, it was reported that $\mathrm{TNF} \alpha$ blockade overcomes resistance to anti-PD-1 in experimental melanoma 
because TNF blockade prevents anti-PD-1-induced TIL cell death as well as PD-L1 and TIM-3 expression. Furthermore, TNF expression positively correlates with expression of PD-L1 and TIM-3 in human melanoma specimens. ${ }^{53}$ More interestingly, prophylactic blockade of human TNF improved colitis and hepatitis in xenografted mice with human colon cancer and moreover, immunotherapeutic control of xenografted tumors was obtained. $^{54}$

Collectively, given the reassuring safety profile of SI without compromising ICI effectiveness, resumption of ICI under secondary prevention with the most appropriate SI may have merit, in particularly if ICI is the only effective anticancer option. We recognize that ICI rechallenge may be considered in specific scenarios with very close follow-up after a very careful and thoughtful balance of risks and benefits in an individualized setting by a multispecialty experienced team, taking into account a plethora of factors, as discussed above.

In general, we advocate for ICI-related colitis/enteritis to resume ICI under VDZ, SS-like irAEs under antiB-cell activating factor \pm anti-CD20 depletion ${ }^{55} 56$; inflammatory arthritis irAEs under $\mathrm{TCZ}^{19}$; hemolytic thrombocytopenia or anemia irAEs under anti-CD20 depletion \pm intravenous immunoglobulin ${ }^{57}$; ICI-related hypereosinophilic syndrome under anti-IL5(R) ${ }^{58}$; ; ICI-related pneumonitis, hepatitis and AIK under TCZ (figure 1). If patients experience new or recurrent high-grade irAEs despite concomitant SI, we advocate definitive ICI discontinuation and consideration of prompt local expertize for appropriate treatment. Ideally, such strategies should be performed as part of well-designed prospective randomized trials or at least the data from these patients should be prospectively collected into databases and registries for subsequent rigorous analyzes.

\section{Author affiliations \\ ${ }^{1}$ Netherlands Cancer Institute, Division of Medical Oncology, Plesmanlaan 121, 1066 CX Amsterdam, The Netherlands \\ ${ }^{2}$ Roswell Park Comprehensive Cancer Center, Elm \& Carlton Streets, Buffalo, New York 14263, USA \\ ${ }^{3}$ Department of Gastroenterology, Hepatology \& Nutrition, University of Texas MD Anderson Cancer Center, Houston, TX 77030, USA \\ ${ }^{4}$ Melanoma Institute Australia and The University of Sydney, Sydeny, New South Wales, Australia \\ ${ }^{5}$ Royal North Shore and Mater Hospitals, Sydney, New South Wales, Australia \\ ${ }^{6}$ University of Washington, Seattle Cancer Care Alliance, Fred Hutchinson Cancer Research Center, Seattle, Washington, USA \\ ${ }^{7}$ Royal Marsden NHS Foundation Trust, London, UK \\ ${ }^{8}$ Centre Hospitalier Universitaire Vaudois (CHUV), Department of Oncology, rue du Bugnon 46, CH-1011 Lausanne, Switzerland \\ ${ }^{9}$ Centre Hospitalier Universitaire Vaudois (CHUV), Department of Medicine, Service of Immunology and Allergy, rue du Bugnon 46, $\mathrm{CH}-1011$ Lausanne, Switzerland ${ }^{10}$ Centre Hospitalier Universitaire Vaudois (CHUV), Vaccine and Immunotherapy Center, rue du Bugnon 17, CH-1011 Lausanne, Switzerland}

Contributors $\mathrm{MO}$ conceived the review and treatment algorithms, $\mathrm{MO}$ and $\mathrm{JH}$ wrote the manuscript and prepared the tables. All authors wrote, commented on and corrected the manuscript.

Funding The authors have not declared a specific grant for this research from any funding agency in the public, commercial or not-for-profit sectors.
Competing interests M0 received education grants from Leenaards Foundation. ME and JT declare no conflicts of interest. SP received education grants, provided consultation, attended advisory boards and/or provided lectures for the following organizations: Amgen, AstraZeneca, Boehringer-Ingelheim, Bristol-Myers Squibb, Clovis, Eli Lilly, F. Hoffmann-La Roche, Janssen, Merck Sharp and Dohme, Novartis, Merck Serono, Pfizer, Regeneron and Takeda. JH received research grants from Novartis, MSD, BMS and Neon Therapeutics, and provided consultation, lectures or attended advisory boards for Amgen, AstraZeneca, Bayer, BMS, Celsius Therapeutics, Gadeta, GSK, Immunocore, MSD, Merck Serono, Neon Therapeutics, Pfizer, Roche/Genentech, Sanofi and Seattle Genetics. YW serves as consultant for Tillotts Pharma. JL received institutional research support for: BMS, MSD, Novartis, Pfizer, Achilles Therapeutics, Roche, Nektar Therapeutics, Covance, Immunocore, Pharmacyclics, Aveo, NIHR RM/ICR Biomedical Research Center for cancer and consultancy from: Achilles, AZ, Boston Biomedical, BMS, Eisai, EUSA Pharma, GSK, Ipsen, Imugene, Incyte, iOnctura, Kymab, Merck Serono, MSD, Nektar, Novartis, Pierre Fabre, Pfizer, Roche / Genentech, Secarna, Vitaccess. PG provided unrelated consultation and advisory board (within 2 years) for Merck, Bristol-Myers Squibb, AstraZeneca, Clovis Oncology, EMD Serono, Seattle Genetics, Foundation Medicine, Driver, Pfizer, QED Therapeutics, HERON Therapeutics, Janssen, Bayer, Genzyme, Mirati Therapeutics, Exelixis, Roche, Glaxo Smith Kline. AM advisory board member for BMS, MSD, Novartis, Roche, Pierre-Fabre.

\section{Patient consent for publication Not required.}

Provenance and peer review Not commissioned; externally peer reviewed.

Open access This is an open access article distributed in accordance with the Creative Commons Attribution Non Commercial (CC BY-NC 4.0) license, which permits others to distribute, remix, adapt, build upon this work non-commercially, and license their derivative works on different terms, provided the original work is properly cited, appropriate credit is given, any changes made indicated, and the use is non-commercial. See http://creativecommons.org/licenses/by-nc/4.0/.

\section{ORCID iD}

Yinghong Wang http://orcid.org/0000-0002-5148-6130

\section{REFERENCES}

1 Blank CU, Rozeman EA, Fanchi LF, et al. Neoadjuvant versus adjuvant ipilimumab plus nivolumab in macroscopic stage III melanoma. Nat Med 2018;24:1655-61.

2 Rozeman EA, Menzies AM, van Akkooi ACJ, et al. Identification of the optimal combination dosing schedule of neoadjuvant ipilimumab plus nivolumab in macroscopic stage III melanoma (OpACIN-neo): a multicentre, phase 2, randomised, controlled trial. Lancet Oncol 2019;20:948-60.

3 Huang AC, Orlowski RJ, Xu X, et al. A single dose of neoadjuvant PD-1 blockade predicts clinical outcomes in resectable melanoma. Nat Med 2019;25:454-61.

4 Forde PM, Chaft JE, Smith KN, et al. Neoadjuvant PD-1 blockade in resectable lung cancer. N Engl J Med 2018;378:1976-86.

5 Martins F, Sofiya L, Sykiotis GP, et al. Adverse effects of immunecheckpoint inhibitors: epidemiology, management and surveillance. Nat Rev Clin Oncol 2019;16:563-80.

6 Santini FC, Rizvi H, Plodkowski AJ, et al. Safety and efficacy of Retreating with immunotherapy after immune-related adverse events in patients with NSCLC. Cancer Immunol Res 2018;6:1093-9.

7 Menzies AM, Johnson DB, Ramanujam S, et al. Anti-Pd-1 therapy in patients with advanced melanoma and preexisting autoimmune disorders or major toxicity with ipilimumab. Ann Oncol 2017;28:368-76.

8 Bowyer S, Prithviraj P, Lorigan P, et al. Efficacy and toxicity of treatment with the anti-CTLA-4 antibody ipilimumab in patients with metastatic melanoma after prior anti-PD-1 therapy. Br J Cancer 2016;114:1084-9.

9 Weber JS, Gibney G, Sullivan RJ, et al. Sequential administration of nivolumab and ipilimumab with a planned switch in patients with advanced melanoma (CheckMate 064): an open-label, randomised, phase 2 trial. Lancet Oncol 2016;17:943-55.

10 Pollack $\mathrm{MH}$, Betof $\mathrm{A}$, Dearden $\mathrm{H}$, et al. Safety of resuming antiPD-1 in patients with immune-related adverse events (irAEs) during combined anti-CTLA-4 and anti-PD1 in metastatic melanoma. Ann Oncol 2018;29:250-5.

11 Simonaggio A, Michot JM, Voisin AL, et al. Evaluation of Readministration of immune checkpoint inhibitors after immunerelated adverse events in patients with cancer. JAMA Oncol 2019;5:1310. 
12 Abu-Sbeih H, Ali FS, Naqash AR, et al. Resumption of immune checkpoint inhibitor therapy after immune-mediated colitis. JCO 2019;37:2738-45.

13 Simonaggio A, Michot JM, Voisin AL, et al. Evaluation of Readministration of immune checkpoint inhibitors after immunerelated adverse events in patients with cancer. JAMA Oncol 2019 doi:10.1001/jamaoncol.2019.1022. [Epub ahead of print: 06 Jun 2019]

14 Abu-Sbeih H, Ali FS, Naqash AR, et al. Resumption of immune checkpoint inhibitor therapy after immune-mediated colitis. J Clin Oncol 2019;37:JCO1900320.

15 Badran YR, Cohen JV, Brastianos PK, et al. Concurrent therapy with immune checkpoint inhibitors and TNF $\alpha$ blockade in patients with gastrointestinal immune-related adverse events. J Immunother Cancer 2019;7:226.

16 Hughes MS, Molina GE, Chen ST, et al. Budesonide treatment for microscopic colitis from immune checkpoint inhibitors. J Immunother Cancer 2019;7:292.

17 Verheijden RJ, May AM, Blank CU, et al. Association of anti-TNF with decreased survival in steroid refractory ipilimumab and Anti-PD1Treated patients in the Dutch melanoma treatment registry. Clin Cancer Res 2020;26:2268-74.

18 Abu-Sbeih H, Ali FS, Wang X, et al. Early introduction of selective immunosuppressive therapy associated with favorable clinical outcomes in patients with immune checkpoint inhibitor-induced colitis. J Immunother Cancer 2019;7:93.

19 Kim ST, Tayar J, Trinh VA, et al. Successful treatment of arthritis induced by checkpoint inhibitors with tocilizumab: a case series. Ann Rheum Dis 2017;76:2061-4.

20 Martins F, Sykiotis GP, Maillard M, et al. New therapeutic perspectives to manage refractory immune checkpoint-related toxicities. Lancet Oncol 2019;20:e54-64.

21 Das S, Johnson DB. Immune-Related adverse events and anti-tumor efficacy of immune checkpoint inhibitors. J Immunother Cancer 2019;7:306.

22 Indini A, Di Guardo L, Cimminiello C, et al. Immune-Related adverse events correlate with improved survival in patients undergoing antiPD1 immunotherapy for metastatic melanoma. J Cancer Res Clin Oncol 2019;145:511-21.

23 Xing P, Zhang F, Wang G, et al. Incidence rates of immune-related adverse events and their correlation with response in advanced solid tumours treated with NIVO or NIVO+IPI: a systematic review and meta-analysis. J Immunother Cancer 2019;7:341.

24 Abu-Sbeih H, Ali FS, Qiao W, et al. Immune checkpoint inhibitorinduced colitis as a predictor of survival in metastatic melanoma. Cancer Immunol Immunother 2019;68:553-61.

25 Sato K, Akamatsu H, Murakami E, et al. Correlation between immune-related adverse events and efficacy in non-small cell lung cancer treated with nivolumab. Lung Cancer 2018;115:71-4.

26 Ricciuti B, Genova C, De Giglio A, et al. Impact of immune-related adverse events on survival in patients with advanced non-smal cell lung cancer treated with nivolumab: long-term outcomes from a multi-institutional analysis. J Cancer Res Clin Oncol 2019;145:479-85.

27 Owen DH, Wei L, Bertino EM, et al. Incidence, risk factors, and effect on survival of immune-related adverse events in patients with nonsmall-cell lung cancer. Clin Lung Cancer 2018;19:e893-900.

28 Suresh K, Naidoo J, Lin CT, et al. Immune checkpoint immunotherapy for non-small cell lung cancer: benefits and pulmonary toxicities. Chest 2018;154:1416-23.

29 Maher VE, Fernandes LL, Weinstock C, et al. Analysis of the association between adverse events and outcome in patients receiving a programmed death protein 1 or programmed death ligand 1 antibody. J Clin Oncol 2019;37:2730-7.

30 Horvat TZ, Adel NG, Dang T-O, et al. Immune-Related adverse events, need for systemic immunosuppression, and effects on survival and time to treatment failure in patients with melanoma treated with ipilimumab at Memorial Sloan Kettering cancer center. $J$ Clin Oncol 2015;33:3193-8.

31 Somasundaram R, Zhang G, Fukunaga-Kalabis $M$, et al. TumorAssociated B-cells induce tumor heterogeneity and therapy resistance. Nat Commun 2017;8:607.

32 Fang D, Nguyen TK, Leishear K, et al. A tumorigenic subpopulation with stem cell properties in melanomas. Cancer Res 2005;65:9328-37.

33 Schlaak M, Schmidt P, Bangard C, et al. Regression of metastatic melanoma in a patient by antibody targeting of cancer stem cells. Oncotarget 2012;3:22-30.

34 Damsky W, Jilaveanu L, Turner N, et al. B cell depletion or absence does not impede anti-tumor activity of PD-1 inhibitors. J Immunother Cancer 2019;7:153.
35 Matson DR, Accola MA, Rehrauer WM, et al. Fatal myocarditis following treatment with the PD-1 inhibitor nivolumab. J Forensic Sci 2018;63:954-7.

36 Johnson DB, Balko JM, Compton ML, et al. Fulminant myocarditis with combination immune checkpoint blockade. N Engl J Med 2016;375:1749-55

37 Zen Y, Yeh MM. Hepatotoxicity of immune checkpoint inhibitors: a histology study of seven cases in comparison with autoimmune hepatitis and idiosyncratic drug-induced liver injury. Mod Pathol 2018;31:965-73.

38 Belliere J, Meyer N, Mazieres J, et al. Acute interstitial nephritis related to immune checkpoint inhibitors. $\mathrm{Br} J$ Cancer 2016;115:1457-61.

39 Marco T, Anna P, Annalisa T, et al. The mechanisms of acute interstitial nephritis in the era of immune checkpoint inhibitors in melanoma. Ther Adv Med Oncol 2019;11:1758835919875549.

40 Suresh K, Naidoo J, Zhong Q, et al. The alveolar immune cell landscape is dysregulated in checkpoint inhibitor pneumonitis. J Clin Invest 2019;130:4305-15.

41 Horisberger A, La Rosa S, Zurcher J-P, et al. A severe case of refractory esophageal stenosis induced by nivolumab and responding to tocilizumab therapy. J Immunother Cancer 2018;6:156

42 Fisher DT, Appenheimer MM, Evans SS. The two faces of IL-6 in the tumor microenvironment. Semin Immunol 2014:26:38-47.

43 Li B, Jones LL, Geiger TL. II-6 promotes T cell proliferation and expansion under inflammatory conditions in association with lowlevel ROR yt expression. J Immunol 2018;201:2934-46.

44 Tsukamoto H, Fujieda K, Miyashita A, et al. Combined blockade of IL6 and PD-1/PD-L1 signaling abrogates mutual regulation of their immunosuppressive effects in the tumor microenvironment. Cancer Res 2018;78:5011-22.

45 Mace TA, Shakya R, Pitarresi JR, et al. II-6 and PD-L1 antibody blockade combination therapy reduces tumour progression in murine models of pancreatic cancer. Gut 2018;67:320-32.

46 Ohno Y, Toyoshima Y, Yurino $\mathrm{H}$, et al. Lack of interleukin-6 in the tumor microenvironment augments type-1 immunity and increases the efficacy of cancer immunotherapy. Cancer Sci 2017;108:1959-66.

47 Ramesh R, Kozhaya L, McKevitt K, et al. Pro-Inflammatory human Th17 cells selectively express $\mathrm{P}$-glycoprotein and are refractory to glucocorticoids. J Exp Med 2014;211:89-104.

48 Ridker PM, MacFadyen JG, Thuren T, et al. Effect of interleukin-1 $\beta$ inhibition with canakinumab on incident lung cancer in patients with atherosclerosis: exploratory results from a randomised, double-blind, placebo-controlled trial. Lancet 2017;390:1833-42.

49 Sandborn WJ, Feagan BG, Rutgeerts P, et al. Vedolizumab as induction and maintenance therapy for Crohn's disease. $N$ Engl J Med 2013;369:711-21.

50 Feagan BG, Rutgeerts P, Sands BE, et al. Vedolizumab as induction and maintenance therapy for ulcerative colitis. N Engl J Med 2013;369:699-710.

51 Munger JS, Huang X, Kawakatsu $\mathrm{H}$, et al. The integrin alpha v beta 6 binds and activates latent TGF beta 1: a mechanism for regulating pulmonary inflammation and fibrosis. Cell 1999;96:319-28.

52 Soler D, Chapman T, Yang L-L, et al. The binding specificity and selective antagonism of vedolizumab, an anti-alpha4beta7 integrin therapeutic antibody in development for inflammatory bowel diseases. J Pharmacol Exp Ther 2009;330:864-75.

53 Bertrand F, Montfort A, Marcheteau E, et al. Tnf $\alpha$ blockade overcomes resistance to anti-PD-1 in experimental melanoma. Nat Commun 2017:8:2256.

54 Perez-Ruiz E, Minute L, Otano I, et al. Prophylactic TNF blockade uncouples efficacy and toxicity in dual CTLA-4 and PD-1 immunotherapy. Nature 2019;569:428-32.

55 Ramos-Casals M, Brito-Zerón P, Bombardieri S, et al. EULAR recommendations for the management of Sjögren's syndrome with topical and systemic therapies. Ann Rheum Dis 2020;79:3-18.

56 Ghosn J, Vicino A, Michielin O, et al. A severe case of neuroSjögren's syndrome induced by pembrolizumab. J Immunother Cancer 2018;6:110.

57 Leaf RK, Ferreri C, Rangachari D, et al. Clinical and laboratory features of autoimmune hemolytic anemia associated with immune checkpoint inhibitors. Am J Hematol 2019;94:563-74.

58 Rothenberg ME, Klion AD, Roufosse FE, et al. Treatment of patients with the hypereosinophilic syndrome with mepolizumab. $N$ Engl $J$ Med 2008;358:1215-28.

59 Kuang FL, Legrand F, Makiya M, et al. Benralizumab for PDGFRA-Negative Hypereosinophilic Syndrome. N Engl J Med 2019;380:1336-46 
60 Larkin J, Chiarion-Sileni V, Gonzalez R, et al. Combined nivolumab and ipilimumab or monotherapy in untreated melanoma. $N$ Engl J Med 2015;373:23-34.

61 Robert C, Schachter J, Long GV, et al. Pembrolizumab versus ipilimumab in advanced melanoma. N Engl J Med 2015;372:2521-32.

62 Motzer RJ, Tannir NM, McDermott DF, et al. Nivolumab plus ipilimumab versus sunitinib in advanced renal-cell carcinoma. $N$ Engl J Med 2018;378:1277-90.

63 Motzer RJ, Escudier B, McDermott DF, et al. Nivolumab versus everolimus in advanced renal-cell carcinoma. $N$ Engl J Med 2015;373:1803-13.

64 Mok TSK, Wu Y-L, Kudaba I, et al. Pembrolizumab versus chemotherapy for previously untreated, PD-L1-expressing, locally advanced or metastatic non-small-cell lung cancer (KEYNOTE-042): a randomised, open-label, controlled, phase 3 trial. Lancet 2019;393:1819-30.

65 Bowyer S, Prithviraj P, Lorigan P, et al. Efficacy and toxicity of treatment with the anti-CTLA-4 antibody ipilimumab in patients with metastatic melanoma after prior anti-PD-1 therapy. Br J Cancer 2016;114:1084-9.

66 Delaunay M, Cadranel J, Lusque A, et al. Immune-checkpoint inhibitors associated with interstitial lung disease in cancer patients. Eur Respir J 2017;50. doi:10.1183/13993003.00050-2017. [Epub ahead of print: 10 Aug 2017].

67 Abu-Sbeih H, Ali FS, Naqash AR, et al. Resumption of immune checkpoint inhibitor therapy after immune-mediated colitis. J Clin Oncol 2019;37:2738-45. 